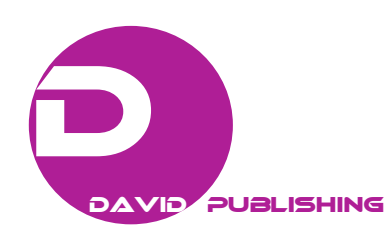

\title{
The Flexible Housing: Criteria and Strategies for Implementation of the Flexibility
}

\author{
Cristiana Cellucci and Michele Di Sivo \\ Architecture Department, University “G. d’Annunzio” of Chieti and Pescara, Pescara 65122, Italy
}

\begin{abstract}
The design of housing systems is today challenged by a highly uncertain context, dominated by the rapid development of functional and technological obsolescence in inherited housing models. If flexibility is the ability of a system to be easily modified and to respond to changes in the environment timely and conveniently, it can be considered as the antidote to obsolescence or the characteristic of the system that guarantees slippage over time. Our paper focuses on the concept of flexibility as a fundamental prerequisite for residential building in order to extend its life cycle design, through strategies and constructive solutions that ensure both the convertibility of the space in response to changing usage and the use of building materials that encourage the reversibility and the long-term easy maintenance of the technological choices that have been implemented. Flexibility is examined both from a conceptual point of view, so as to obtain a clear and logical definition that is distinct from related terms, as well as from a practical point of view, by finding ways to incorporate this requirement into the designing of housing.
\end{abstract}

Key words: Resilience, spatial flexibility, technological flexibility, reversibility.

\section{Introduction}

Some of the main problems affecting social housing in the last decades have been the disposal and renovation of these inherited buildings, as well as the risk of becoming technically or functionally obsolete in the short term, because the performance of the buildings no longer has a competitive edge in the housing market nor they satisfy the needs of the users.

This inability to manage the uncertainties of the socio-economic context and the various needs that arise from different types of social housing usage tends to render the housing system inadequate and reduce its shelf life.

This suggests the need to rethink the concepts of obsolescence, life span and flexibility during the design phase in general and particularly during the renovation works. If flexibility is defined as the ability of a system to be easily modified and to respond to user needs in a timely and effective manner, then it

Corresponding author: Cristiana Cellucci, Ph.D., research fields: social and political aspects of architecture and the built environment. E-mail: cristiana.cellucci@gmail.com. can be considered an antidote to obsolescence and a characteristic of the system that ensures the extension of its life cycle over time.

For this reason, in the context of our research, we have adopted the concept of flexibility as a characteristic of a system that is able to:

- manage the uncertainty of the environment in which the system operates in the short term, allowing the system to respond to user needs that may change during the operational phase and which result in the inadequacy of the initial objectives, the design requirements, and in terms of the performance and methods used to meet needs in a timely and effective manner [1];

- set up and direct the building's rhythm of change in the long term, by making it able to reorganize and innovate itself against external disturbances [1].

The research proposes the project strategies aimed at ensuring the survival over time of the building, thanks to the ability to implement several cycles of use of body building, confront the ability to reconfigure the internal structure and intervene in a simplified way on the technological system that 
governs the space.

\section{Flexibility and Resilience}

By analyzing the use of the term of flexibility in other fields of study, in particular in production systems and complex engineering systems, such as aerospace engineering, it becomes clear that today flexibility takes on a role that is similar to the concept of quality about 20 years ago: namely, one that is vague and difficult to understand, but is fundamental to the competitiveness and survival of the system [2].

The study of complex systems shows a close relationship between time, uncertainty, flexibility and resilience. Typically, a system progresses through a life cycle characterized by phases of growth, maturity, decline, and then "dies" (ceases to be useful) due to the process of functional and technological obsolescence, generally caused by the inability to manage uncertainty (Fig. 1).

Out of all the structures and artifacts of antiquity, only a small residual part has survived until today, reminding us of the transience of human endeavor. In modern times, industrial products, infrastructure and objects have an ephemeral relationship with time, due to the impermanence of the artifacts. These now familiar technological objects are the obvious warning that nothing is permanent. Through physical and functional decay or the loss of economic viability, the "hands of time bear down on the knowledge" of mankind [3]. Uncertainty, understood as the absence of knowledge of a system's possible evolution [2], normally has negative connotations. A key point, in the theory of the economist Stigler [4], is the link between flexibility and uncertainty. Flexibility is needed to address uncertainty, which can be caused by various factors: a change in demand, changes in the user needs, innovative technology, new regulations or the availability of resources [5]. Shi and Daniels [6] consider flexibility to be "a cover against uncertainties, as a direct consequence of a general complexity due to technological progress and to changing user needs”.

In this regard, Darwin's [7] theory of the selection of species or the economist Terborgh's [8] deliberations on the lifespan of instrumental assets [1] has shown that there are living organisms and human artifacts which are more able to adapt to life's changing environments, as opposed to rigid biological or technological systems that are incapable of reacting to change. In other words, flexibility reduces a system's exposure to uncertainty and it is the solution that mitigates against the risks derived from the accelerated evolution of the context-risks associated with technological obsolescence. Flexibility is therefore the property that makes the system resilient and capable of absorbing shocks and/or environmental disturbances without undergoing major alterations in its functional organization, structure or identifying characteristics [9].

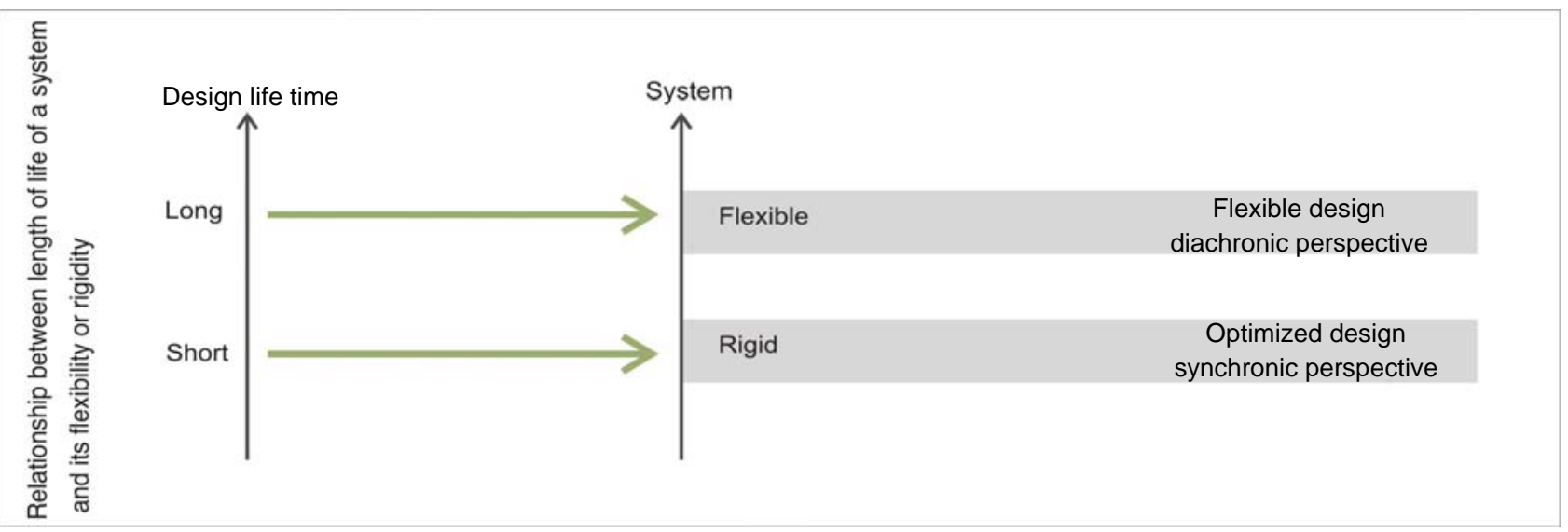

Fig. 1 Relationship between length of life of a system and its flexibility or rigidity. 


\section{Classification, Criteria and Strategies of Approaches to Flexibility}

Throughout history, flexibility has been a central theme in the design of a dwelling. Sometimes it has been implemented within a typological framework, giving the dwelling versatility and internal convertibility through the adaptability of the internal layout. On other occasions, flexibility is achieved from a technological point of view, through flexible networks and terminals, which can be physically moved and repositioned and which are conceived with their easy maintenance in mind. In other cases, flexibility is achieved through morphological-structural choices and building technologies that allow building components to be reversed and interchanged.

Four main tendencies born from specific needs can be identified, and subsequently evolved over time: spatial flexibility in a fixed surface area; evolutionary spatial flexibility; technological flexibility related to construction techniques; technological flexibility related to the easy maintenance of the installations and building sub-systems.

In the four analyzed approaches, flexibility is applied in order to mitigate against specific forms of uncertainty (e.g., variability of intended usage, variability of uses, etc.), which is sometimes more related to a spatial-typological aspect and sometimes more related to a technical theme. So, even if concrete examples of the application of flexibility requirements in social housing design have been found in this field of literature, a synchronic angle in the design of a house's spaces emerges, which tends to optimize the features of a house according to the socio-cultural context of a given period. This type of approach seems more reminiscent of the theories of robust design, universal design and optimized design rather than that of flexible design. There is, on the other hand, no consideration of timeframes that would introduce a diachronic angle to the design of the house.

For each of the four tendencies, we identified strategies that are designed to provide flexibility and space technology. A list of these strategies that can be combined to ensure a high level of flexibility in the design of the house are as follows.

\subsection{Spatial Flexibility in a Fixed Surface Area}

This consists of the study of possible design strategies capable of conferring high internal convertibility without modifying the total volume of the building. In the design of residential buildings, this results in the prevision of interface spaces that can be assigned different functions over time and also results in the set up of technical systems and installations which are compatible with possible changes in the distributive layouts [10-12] .This type of flexibility is achieved by means of equipped technical zones contained in very small polyfunctional spaces or by means of fixed or mobile technical nuclei within a single flexible space (Fig. 2).

\subsubsection{Strategy: Redundancy Access}

This strategy provides the option to decrease the size of the dwelling at any given time, when the household no longer needs the entire surface area of the house and can divide it into two or more units, or can assign part of the surface area to another dwelling or activity (an office). The advance planning of a minimum of two access points (depending on the initial size of the house) can have a significant impact on the overall cost attached to any eventual division of the dwelling into two units.

3.1.2 Strategy: Customizing Privacy Needs and Social Needs

This strategy seeks to create an equal balance between the privacy needs and the social needs of the household, through the organization of a space that guarantees the seclusion of the living units of the bedroom, bathroom and study, and the sharing of the living units where the cohabitants socialize. This affects both the arrangement of the living units and the 


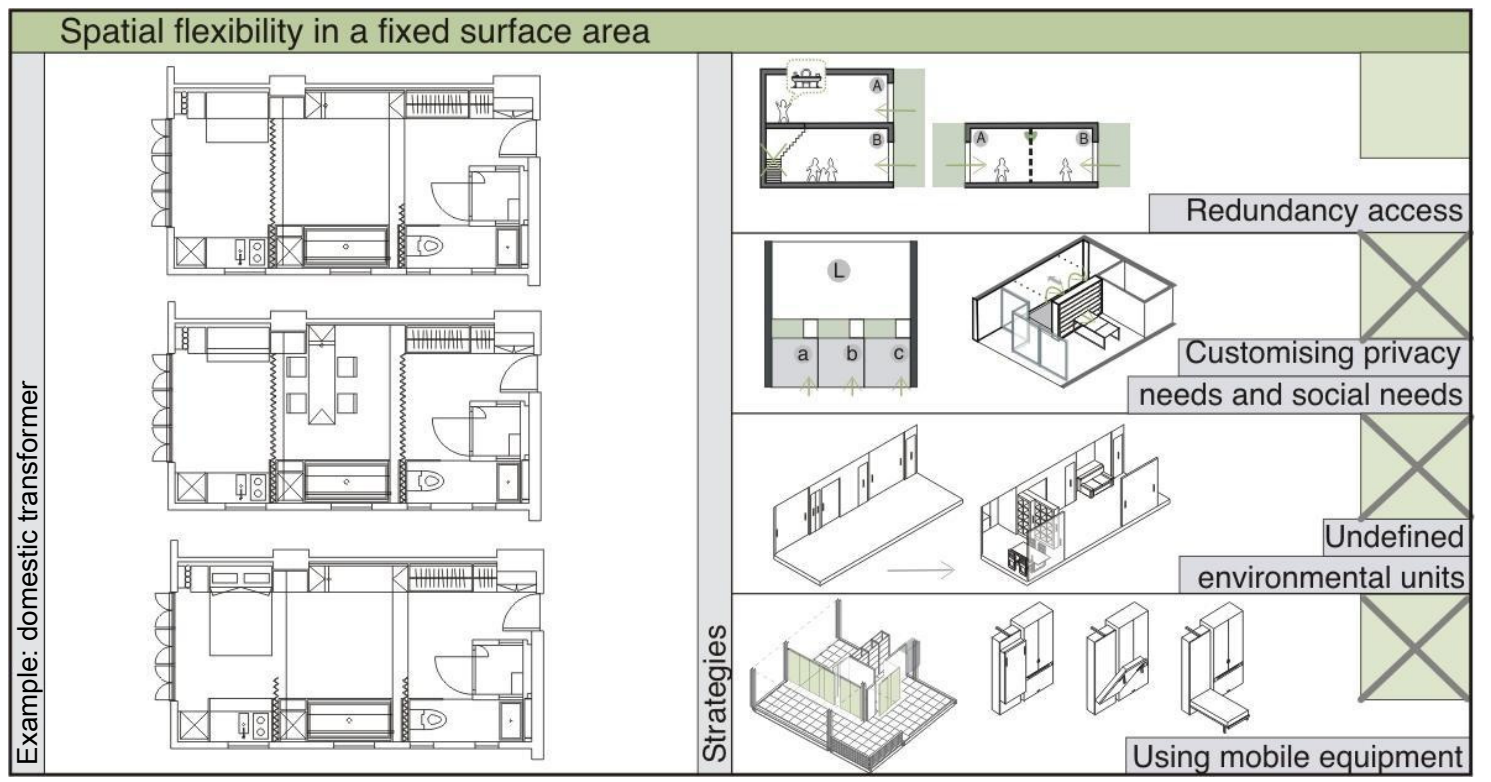

Fig. 2 Domestic transformer, Gar Chang, Hong Kong, China, 2007.

systems that ensure their comfort: lighting elements for the modification of light and heat, equipment and moveable walls that ensure the size of the rooms match their function, etc..

\subsubsection{Strategy: Undefined Environmental Units}

This strategy is based on equipping the house with undefined spaces that can adapt their usage without having to physically change. This is possible either through the neutral sizing of the living units in order to accommodate any function and the elimination of hierarchy between them (whereby a bedroom can convert to a study, etc.), or through the conception of the space as a universal container where adaptable living units are organized by moving walls or furniture (wardrobes, bookcases, etc.).

\subsubsection{Strategy: Using Mobile Equipment.}

This strategy allows the spatial and functional reorganization of the entire housing unit with quick turnaround, thanks to the displacement of equipped walls, furniture cabinets or prefabricated modular interior partitions are mounted with dry joints. This solution is effective especially in small spaces, without partitions, where the partitioning is done through the use of mobile equipment which enables the user to use the same surface differently.

\subsection{Evolutionary Spatial Flexibility}

This consists of programming the life cycle of the house and its grades of convertibility with alternating phases of expansion and contraction based on changing user needs. This therefore requires spatial strategies and complex technological systems. The idea of convertibility connects delimitating surface areas and structural logic through the increase in the total volume of the building. These techniques give the building a flexibility that allows it to change radically and easily, as well as making it possible to completely redesign the spaces and surface areas in a relatively short timeframe and at a low cost (Fig. 3) [12].

3.2.1 Strategy: Increasing the Surface Area within the Existing Support

This strategy allows the increase of the surface of the house through the closure of spaces that are already built and of relevance to the house (support existent), but they are not considered habitable, because they are open, for which there is no need to extend or modify the structure. The new space can be a balcony or a terrace, with small architectural interventions, may be closed to form part of the interior of the house. 


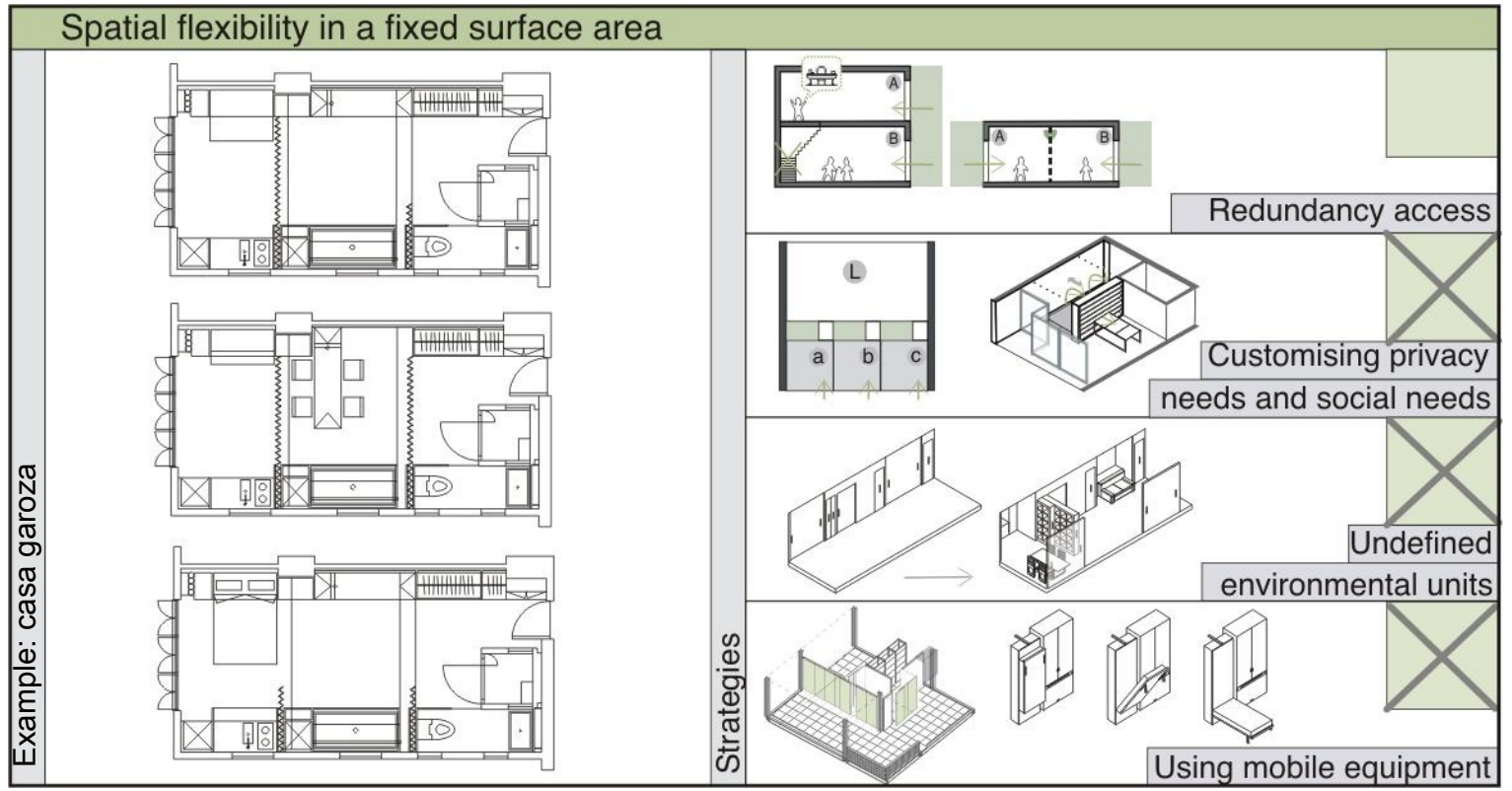

Fig. 3 Casa garoza, Herreros Arquitectos, Spagna, 2010.

3.2.2 Strategy: Increasing the Dwelling's Surface Area in a New Structure and the Increasing of the Initial Volume

This strategy allows the growth of useful surface of the house creating new spaces, compared to the initial volume, over a new support, then through the addition of structural elements on a patio or an external garden. In this case, the house must be designed for modular elements that can be expanded in the three spatial directions according to the need, because planned in designed stage, through the use of prefabricated modular elements.

3.2.3 Strategy: Increasing the Internal Surface Area by the Addition of Living Units

This strategy allows the increase of the useful surface of the housing, without affecting the initial volume because the increase takes place within it. This increase is possible inside of a house on two levels, through the closure of horizontal full-height spaces in favor of environmental new units, such as adding an extra bedroom for the birth of a child.

\subsection{Technological Flexibility Related to Construction Techniques}

This makes it possible to easily convert the home through the use of construction solutions and innovative building components that replace the traditionally fixed parts of the building (partitions, cladding and installations) so that they can be changed, updated or replaced according to the needs of the household. It can be implemented with the use of a system or kit containing light components that are pre-fabricated or partially pre-fabricated and pre-assembled or assembled on site. This makes it possible to manipulate the spaces of a house, leaving the structural parts intact and adding or subtracting construction elements. In this way, the total surface area of the home can by expanded or contracted over time, forming a continuous state of dynamic equilibrium between the building and the household's demands and needs (Fig. 4).

3.3.1 Strategy: Adjustment and Adaptability of the Building Envelope

This strategy allows an upgradable facade of the housing, which usually needs to be rehabilitated every 20 years to technical or esthetic considerations. The adjustment of the envelope can include the possibility of enlarging the internal space within a new or existing structure. Among the possible solutions are the use of mobile facades that roll along a pre-existing 


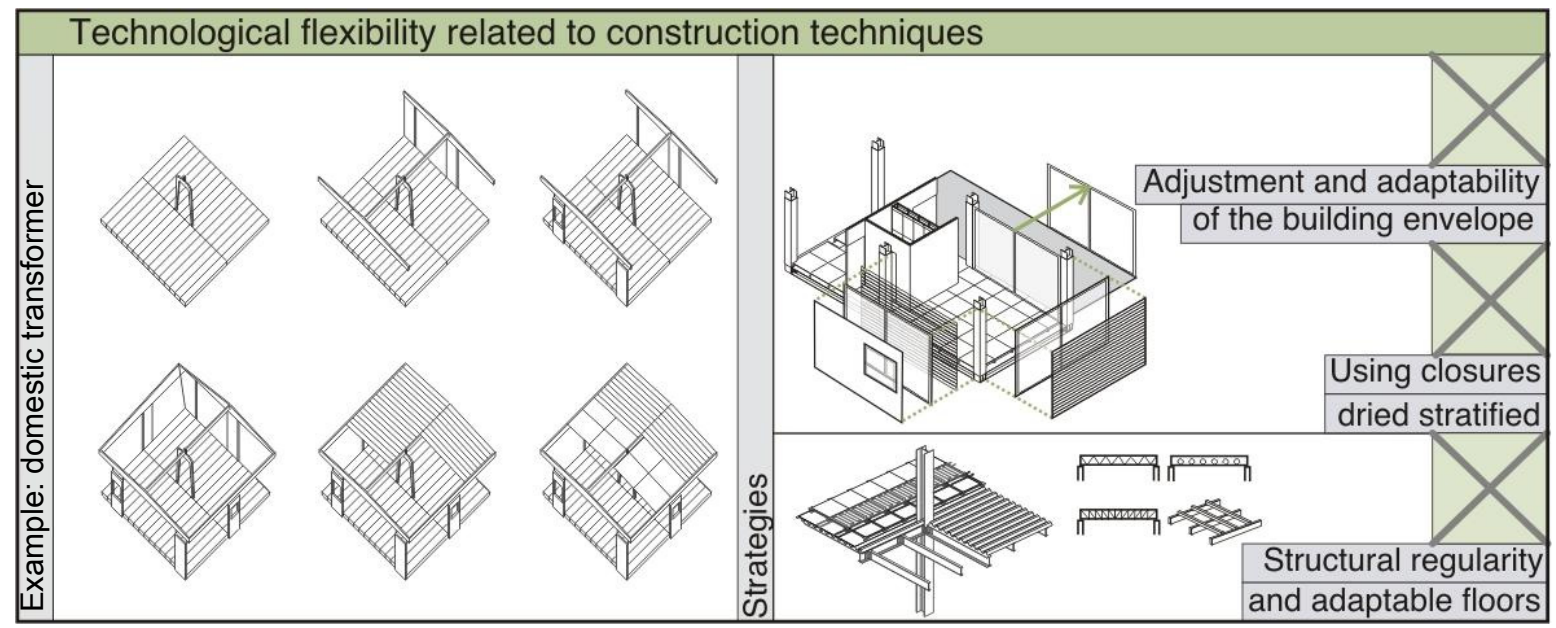

Fig. 4 Tropical house, Jean Prouvè, Francia, 1949.

loggia in order to extend the internal space, and non-load bearing curtain walls that allow some closure elements to be disassembled and then either replaced or subsequently reassembled in a new arrangement. These solutions cost less and take less time than traditional exterior walls.

\subsubsection{Strategy: Using Closures Dried Stratified}

This strategy allows the housing reversibility with deadlines fast enough and reuses part or all of the components used. This strategy allows, therefore, a total housing reversibility, the replacement of some components with others that provide higher performance and both the change of position of the same (seals or partitions walls). The closures, vertical opaque or transparent, can be thought of as packages stratified dry united with mechanical junctions without the use of materials of connection (adhesives and sealants), easily removable and replaced.

3.3.3 Strategy: Structural Regularity and Adaptable Floors

This strategy consists of the following: organizing the structural system over a regular grid, which is essential to ensure the easy convertibility of the building; the use of materials that ensure maximum reversibility of the system in addition to the flexibility of the internal spaces, through the disassembly and reassembly of metallic components; and the use of hollow or rectangular beams capable of integrating the installation system inside the structural form, leaving the internal space completely free of constraints that could obstruct its convertibility. It is also important to envisage adequate over-sizing of the supporting structure (taking into account the increase of costs) in anticipation of possible extensions to the property at a later date.

\subsection{Technological Flexibility Related to the Easy Maintenance of the Installations and Building Sub-systems}

This seeks to improve the flexibility of the home by ensuring its maintenance with the easy substitution and integration of old and new technical components, at a low cost and in a short timeframe. On the assumption that an organism may be functionally reused or may evolve various types of use that organism must show a high degree of flexibility in order to minimize the amount of work needed to meet new requirements or create new functions, an example of this can be seen in the layout of installations where the pipelines have been usefully laid out on the perimeter of the building so that they are accessible from the outside. As well as minimizing the interference of maintenance work on the pipeline during the operating phase, this also affords a high level of flexibility with regard to future uses, thanks to the sustainable autonomy of the installation's layout plan (Fig. 5) [13]. 

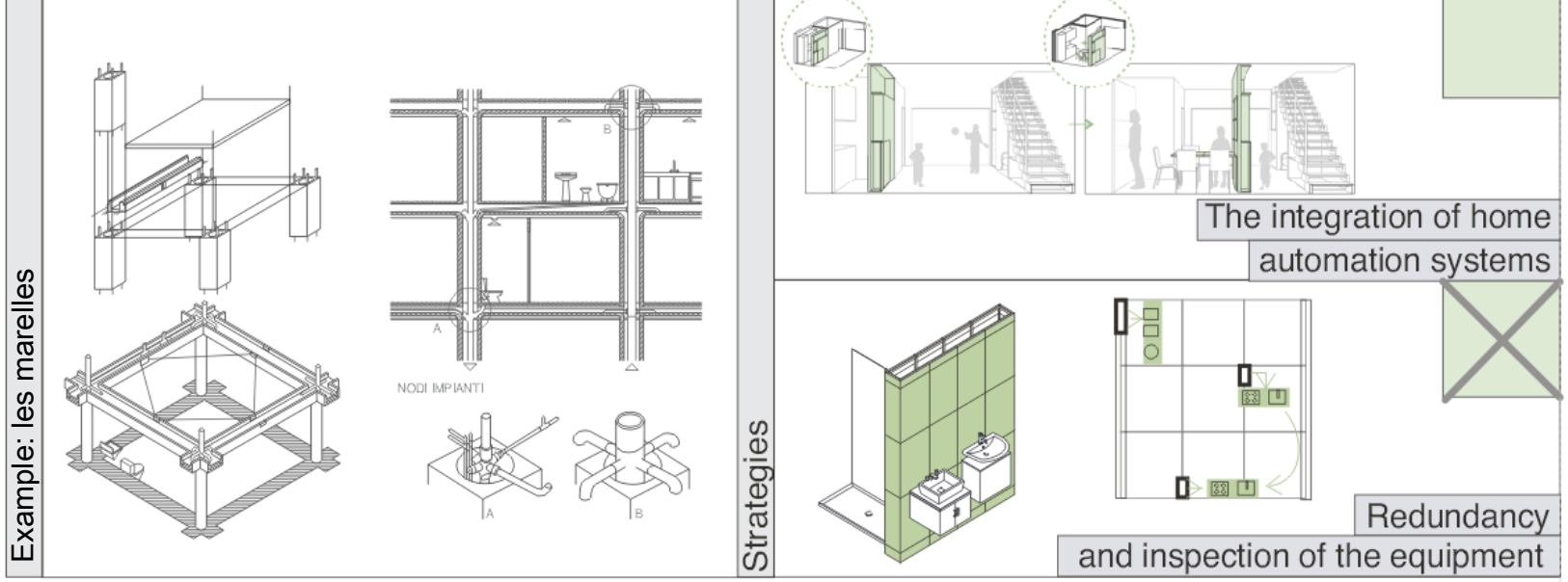

Fig. 5 Les marelles, Bernard Kohn, Georges Maurius, 1971.

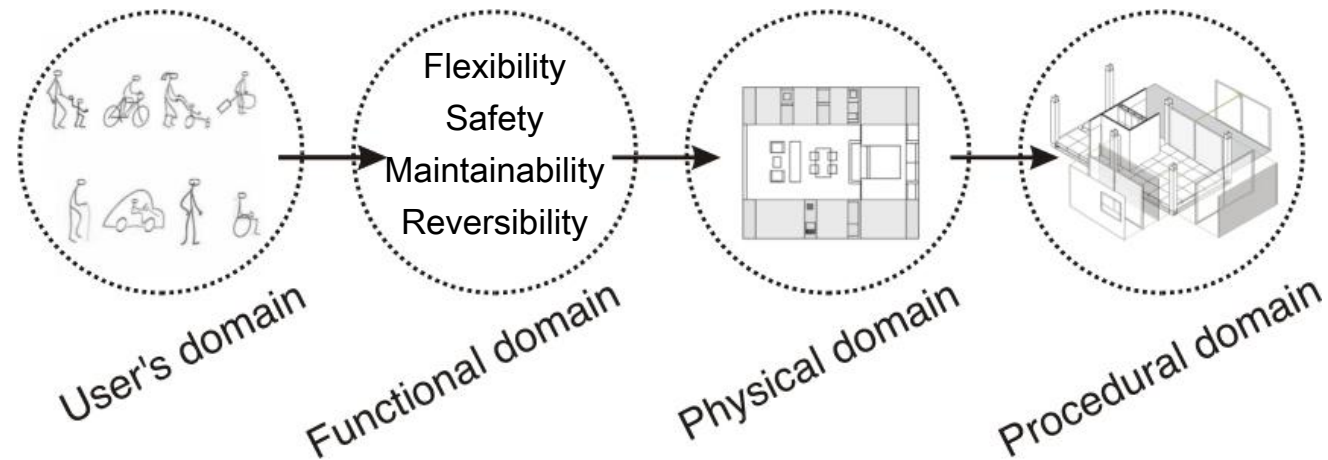

Fig. 6 Four domains of the design.

3.4.1 Strategy: The Integration of Home Automation Systems

This strategy enables the easy convertibility of the space, through the use of home automation systems that make it easier to manage internal changes. These systems can be connected to specific vertical closures, programmed to transform loggias and terraces, thereby increasing the internal space, or they can be connected to a system of storage units capable of transforming the space depending on how they are used. This strategy envisages the programming of feasible spatial solutions and it is particularly useful for certain types of users, such as the disabled or the elderly.

3.4.2 Strategy: Redundancy and Inspection of the Equipment

This strategy consists in downsizing the technological systems in order to allow any changes or additions of nuclei technical (kitchen and bathroom). In the case of refunctionalization, enlargement or reduction, you must be able to reprogram the plant system, possibly implementing it, reducing it or replacing parts without having to change the system. A significant strategy is the planning and scheduling of points of adduction (water and gas), at design time, access function or possible divisions that require adding additional technical areas, etc.

\section{Conclusions}

In order to incorporate the concept of flexibility in designing of housing, the four domains that make up the process of design must be considered: (1) the user's domain, through the analysis of functional and psychological needs and their variations over time; (2) 
the functional domain, which translates needs into design requirements (flexibility, maintainability, reversibility, etc.); (3) the physical domain, linked to the design's spatial and technological choices which satisfy the functional domain (these are the strategies mentioned in the previous section); (4) the procedural domain, through designs that tailor the programmed dimension of the building (to various users, to the need to customize the home), linking it to the rediscovery of low-cost construction systems and local materials, and to the collaboration between local professionals.

The design is, thus, defined as an interplay or a mapping between what we want to achieve and how we want to achieve it. User's domain represents "what we want to achieve", whereas the procedural domain, on the right, represents "how we propose to satisfy what is specified in the user's domain" (Fig. 6):

(1) The terms environment, requirements, performance and methods refer to the definitions of the IEEE Std 1233 Edition;

(2) Time and uncertainty are inherently linked, because, if there were no tomorrow, there would be no uncertainty. Time transforms uncertainty, which in turn has been shaped by the horizon on time: the future is a playing field [14].

\section{Rseferences}

[1] Cellucci, C. 2014. "Time and Resilience: Technological and Spatial Flexibility for the New Home Designing."
Ph.D. thesis, University "G. d'Annunzio" of Chieti and Pescara.

[2] Upton, D. M. 1995. "What Really Makes Factories Flexible?." Harvard Business Review 73 (4): 74-84.

[3] Saleh, J. H., Lamassoure, E. S., Hastings, D. E., and Newman, D. J. 2003. "Flexibility and the Value of On-orbit Servicing: New Customer-Centric Perspective." Journal of Spacecraft and Rockets 40 (2): 279-91.

[4] Stigler, G. 1939. "Production and Distribution in the Short Run." The Journal of Political Economy 47 (3): 305-27.

[5] Sethi, A. K., and Sethi, S. P. 1990. "Flexibility in Manufacturing: A Survey." International Journal of Flexible Manufacturing Systems 2 (4): 289-328.

[6] Shi, D., and Daniels, R. L. 2003. "A Survey of Manufacturing Flexibility.” IBM Systems Journal 2 (3): 414-27.

[7] Darwin, C. 2005. On the Origin of Species. Introduced by Leakey, R. New York: Hill and Wang.

[8] Terborgh, G. 1949. Dynamic Equipment Policy. New York: McGraw-Hill.

[9] UNEP (United Nations Environment Programme). 2005. Climate Change. The Role of Cities. Nairobi: UNEP.

[10] Turchini, G., and Grecchi, M. 2006. Nuovi Modelli per l'Abitare (New model for living). Milano: Il Sole 24 Ore. (in Italian)

[11] Malighetti, L. E. 2008. Progettare la Flessibilità, Tipologie e Tecnologie per la Residenza (Design to Flexibility, Types and Technology for the Home). Milano: Maggioli Editore. (in Italian)

[12] Capolongo, S. 2012. Architecture for Flexibility in Healthcare. Milano: Franco Angeli.

[13] Di Sivo, M., and Ladiana, D. 2009. "Manutenzione e Progetto, Verso un'Etica Della Sostenibilità.” In Antologia della Manutenzione. Milano: Aiman, 1-13. (in Italian)

[14] Bemstein, P. 1996. Against the Gods: The Remarkable Story of Risk. New York: Wiley and Sons. 